\title{
Associação entre condições sistêmicas e gravidade da doença periodontal em pacientes atendidos na Clínica-Escola da UFCG
}

\author{
Association between systemic conditions and the gravity of periodontal \\ disease in patients attended at the UFCG School clinic
}

\author{
Katryne Targino RODRIGUES ${ }^{a} \mathbb{B}^{\mathbb{B}}$, Luanna Abílio Diniz Melquíades de MEDEIROS ${ }^{a}$ (i), \\ João Nilton Lopes de SOUSA ${ }^{a}$ (D), Gêisa Aiane de Morais SAMPAIOa (D), \\ Rachel de Queiroz Ferreira RODRIGUES ${ }^{a *}$ (1)
}

aUFCG - Universidade Federal de Campina Grande, Unidade Acadêmica de Ciências Biológicas, Patos, PB, Brasil

Como citar: Rodrigues KT, Medeiros LADM, Sousa JNL, Sampaio GAM, Rodrigues RQF. Associação entre condições sistêmicas e gravidade da doença periodontal em pacientes atendidos na Clínica-Escola da UFCG. Rev Odontol UNESP. 2020;49:e20200025. https://doi.org/10.1590/1807-2577.02520

\begin{abstract}
Resumo
Introdução: A doença periodontal é uma doença inflamatória crônica dos tecidos de proteção e suporte dos dentes. As doenças ou alterações de ordem sistêmica, como diabetes, alterações cardiovasculares e pulmonares, distúrbios hormonais e outras, não iniciam a doença periodontal, mas podem acelerar uma doença preexistente, aumentando sua progressão e destruição tecidual. Objetivo: 0 presente estudo teve como objetivo avaliar, por meio de prontuários clínicos, uma possível associação entre as condições sistêmicas e a gravidade da doença periodontal em pacientes atendidos na Clínica-Escola de Odontologia da Universidade Federal de Campina Grande, campus CSTR (UFCG-CSTR). Material e método: Para o estudo, foram avaliados 1.035 prontuários clínicos dos pacientes que procuraram atendimento na Clínica-Escola de Odontologia da UFCG-CSTR durante os anos de 2012 a 2017. Resultado: A população estudada apresentou prevalência do sexo masculino $(50,9 \%)$ e diagnóstico de doença gengival $(63,6 \%)$ e periodontal (35,8\%). As condições sistêmicas mais prevalentes foram hipertensão (15\%), diabetes $(7,5 \%)$ e cardiopatias $(5,8 \%)$. Além disso, 20,2\% relataram ser fumantes ou ex-fumantes, enquanto o uso de medicação foi observado em $28,3 \%$ dos casos. Foi verificada associação estatisticamente significativa entre doença periodontal, sexo masculino, faixa etária mais avançada, hipertensão arterial, tabagismo ou histórico de tabagismo e diabetes. Conclusão: Foi observada uma quantidade de dentes igual ou menor do que $10 \mathrm{com}$ maior frequência entre os pacientes com idade igual ou superior a 60 anos, hipertensos, diabéticos, cardiopatas e fumantes ou ex-fumantes, sugerindo, desse modo, uma maior gravidade da doença periodontal nesses indivíduos.
\end{abstract}

Descritores: Doenças periodontais; fatores de risco; ficha clínica.

\begin{abstract}
Introduction: Periodontal disease is a chronic inflammatory disease of the protective and supporting tissues of the teeth. Systemic diseases or changes, such as diabetes, cardiovascular changes, lung changes, hormonal disorders and others, do not start periodontal disease, however they can accelerate a pre-existing disease increasing its progression and tissue destruction. Objective: The present study aimed to evaluated, through clinical records, a possible association between systemic conditions and the severity of periodontal disease in patients seen at the Clinical School of Dentistry, Federal University of Campina Grande, Campus CSTR (UFCG-CSTR). Material and method: The study evaluated 1035 medical records of patients who sought care at the Clinical School of Dentistry of the Federal University of Campina Grande, Campus CSTR during the years 2012 to 2017. Result: The studied population had a prevalence of males (50.9\%) and a diagnosis of gingival and periodontal disease of $63.6 \%$ and $35.8 \%$, respectively. The most prevalent systemic conditions were hypertension (15.0\%), diabetes (7.5\%) and heart disease (5.8\%). About $20.2 \%$ reported being smokers or ex-smokers. The use of medication was observed in $28.3 \%$ of the cases. There
\end{abstract}


was a statistically significant association between periodontal disease, male gender, older age group, arterial hypertension, smoking or history of smoking and diabetes. Conclusion: A number of teeth equal to or less than 10 was observed more frequently among patients aged 60 years or more; hypertensive, diabetic, cardiac patients and smokers or ex-smokers, thus suggesting a greater severity of periodontal disease in these individuals.

Descriptors: Periodontal diseases; risk factors; clinical record.

\section{INTRODUÇÃO}

A doença periodontal (DP) é definida como uma doença inflamatória crônica multifatorial associada com biofilme disbiótico que contém um aglomerado de patógenos anaeróbicos orais envoltos em uma matriz de polissacarídeos, além de ser caracterizada pela destruição progressiva dos tecidos de inserção ${ }^{1,2}$.

De longa data tem sido um consenso em Odontologia que infecções crônicas dos dentes e seus periodontos, mesmo que o paciente não sinta desconforto, podem resultar em lesões orgânicas insidiosas a distância, eventualmente fatais. Um acúmulo de evidências tem levado a "[...] considerar-se as condições bucais não como problemas isolados, mas em suas relações com o corpo humano como um todo"3 (p. 23-24).

O biofilme dentário é o iniciador da DP, no entanto o modo como ele afeta o indivíduo, a manifestação da doença e sua progressão dependem das defesas do hospedeiro. Fatores sistêmicos modificam a periodontite, principalmente, por meio de seus efeitos nas defesas imunológicas e inflamatórias normais. Como exemplos de fatores sistêmicos pode-se citar uma redução no número ou no funcionamento de leucócitos polimorfonucleares (PMNs), que resulta em aumento da taxa e gravidade da destruição periodontal 4 .

A relação de mão dupla entre DP e condições sistêmicas vem sendo investigada ${ }^{2-4}$, levando à definição de medicina periodontal. É bem sabido que a DP pode afetar doenças sistêmicas, e vice-versa ${ }^{5}$.

Portanto, as doenças ou alterações de ordem sistêmica, como diabetes, alterações cardiovasculares e pulmonares, distúrbios hormonais e outras, não iniciam a DP, mas podem acelerar uma doença preexistente, aumentando sua progressão e destruição tecidual6.

Sendo assim, este estudo teve como objetivo avaliar o perfil de pacientes portadores de DP, verificando se há associação entre as condições sistêmicas e a gravidade da DP. Além disso, o presente estudo favorece a criação de meios que possibilitem a redução desses problemas, como o acompanhamento multidisciplinar, buscando uma melhoria na qualidade de vida desses pacientes.

\section{MATERIAL E MÉTODO}

Trata-se de um estudo observacional transversal de caráter retrospectivo com documentação direta de dados qualitativos. A pesquisa foi iniciada somente após aprovação pelo Comitê de Ética em Pesquisa do Hospital Universitário Alcides Carneiro da Universidade Federal de Campina Grande (HUAC/UFCG), com parecer de número: 3.620.802. Tendo em vista que a pesquisa utilizou somente dados obtidos a partir da investigação de prontuários com as informações referentes aos pacientes, foi solicitada a dispensa do Termo de Consentimento Livre e Esclarecido (TCLE).

Para o estudo, foram avaliados 1.035 prontuários clínicos dos pacientes que procuraram atendimento na Clínica-Escola de Odontologia da Universidade Federal de Campina Grande, campus CSTR (UFCG-CSTR), durante os anos de 2012 a 2017. A escolha dos prontuários para avaliação foi realizada aleatoriamente. Foram incluídos na pesquisa apenas prontuários que 
apresentassem dados da propedêutica estomatológica e da clínica de periodontia e excluídos aqueles prontuários cujo preenchimento estivesse incompleto, chegando ao número final de 173 prontuários. Os dados de cada paciente foram anotados em ficha desenvolvida para este estudo, na qual estão presentes dados sobre sexo, idade, número de dentes presentes, condição sistêmica, uso de medicação (anticoncepcionais, anti-hipertensivos, imunossupressores e anticonvulsivantes), diagnóstico periodontal e presença de alterações mucogengivais no periodonto (recessão, hiperplasia, inserção do freio, mobilidade dentária, lesão de furca e presença de mucosa ceratinizada). As alterações no periodonto e condições sistêmicas poderiam estar isoladas ou associadas. Os dados obtidos a partir das fichas de avaliação foram analisados estatisticamente, em que se obtiveram as doenças periodontais mais prevalentes na amostra escolhida, associando-as com alguma condição sistêmica e/ou uso de medicação.

Inicialmente, foi realizada a análise estatística das variáveis objetivando caracterizar a amostra. Foram calculadas as medidas de tendência central e de variabilidade para as variáveis quantitativas, bem como as frequências absolutas e percentuais para as variáveis categóricas. Em seguida, foi empregado o teste qui-quadrado de Pearson (ou teste exato de Fisher quando apropriado) para determinar associação entre a condição periodontal e quantidade de dentes presentes e as demais variáveis investigadas. 0 nível de significância foi fixado em $\mathrm{p}<0,05$. Todas as análises foram realizadas com o auxílio do software SPSS, versão 20.0, considerando um intervalo de confiança de 95\%.

\section{RESULTADO}

A Tabela 1 mostra a distribuição dos casos atendidos de acordo com as características sociodemográficas e condição clínica periodontal. A maioria era do sexo masculino $(50,9 \%)$, tinha entre 20 e 29 anos de idade (28,9\%) e apresentava mais de 20 dentes presentes $(77,5 \%)$. A prevalência de diagnóstico foi de gengivite $(63,6 \%)$ e periodontite $(35,8 \%)$. Os dados também evidenciaram que as alterações periodontais mais frequentes foram recessão gengival $(57,8 \%)$, freio ativo $(16,8 \%)$, mobilidade dentária $(8,1 \%)$, lesão de furca $(2,9 \%)$ e hiperplasia gengival $(1,7 \%)$.

Tabela 1. Distribuição dos casos atendidos de acordo com as características sociodemográficas e condição clínica periodontal

\begin{tabular}{lcc}
\hline \multicolumn{1}{c}{ Variáveis } & $\mathbf{n}$ & \% \\
\hline Sexo & & \\
Feminino & 85 & 49,1 \\
Masculino & 88 & 50,9 \\
Faixa etária & & \\
$\leq 19$ anos & 27 & 15,6 \\
$20-29$ anos & 50 & 28,9 \\
$30-39$ anos & 41 & 23,7 \\
$40-49$ anos & 33 & 19,1 \\
$50-59$ anos & 17 & 9,8 \\
$\geq 60$ anos & 5 & 2,9 \\
No de dentes presentes & & \\
$0-10$ & 9 & 5,2 \\
$11-20$ & 30 & 17,3 \\
$>20$ & 134 & 77,5 \\
Diagnóstico periodontal & & 0,6 \\
Periodonto saudável & 1 & 63,6 \\
Gengivite & 110 & 35,8 \\
Periodontite & 62 & \\
\hline
\end{tabular}


Tabela 1. Continuação...

\begin{tabular}{lcc}
\hline \multicolumn{1}{c}{ Variáveis } & $\mathbf{n}$ & $\mathbf{\%}$ \\
\hline Lesão de furca & 5 & 2,9 \\
Presente & 168 & 97,1 \\
Ausente & & \\
Mobilidade dentária & 14 & 8,1 \\
Presente & 159 & 91,9 \\
Ausente & & \\
Recessão gengival & 100 & 57,8 \\
Presente & 73 & 42,2 \\
Ausente & & \\
Hiperplasia & 3 & 1,7 \\
Presente & 170 & 98,3 \\
Ausente & & 16,8 \\
Freio ativo & 29 & 83,2 \\
Presente & 144 & \\
Ausente & & 99,4 \\
Mucosa ceratinizada & 172 & 0,6 \\
Sim & 1 & $\mathbf{1 0 0 , 0}$ \\
Não & $\mathbf{1 7 3}$ & \\
Total & & \\
\hline
\end{tabular}

De acordo com a Tabela 2, foi constatado que as condições sistêmicas mais prevalentes foram hipertensão (15\%), diabetes (7,5\%) e cardiopatia (5,8\%). Além disso, 20,2\% relataram ser fumantes ou ex-fumantes, enquanto o uso de medicação sistêmica foi observado em $28,3 \%$ dos casos.

Tabela 2. Distribuição dos casos atendidos de acordo com as condições sistêmicas, tabagismo e uso de medicação

\begin{tabular}{lcc}
\hline \multicolumn{1}{c}{ Variáveis } & $\mathbf{n}$ & $\mathbf{\%}$ \\
\hline Hipertensão & 26 & 15,0 \\
Sim & 147 & 85,0 \\
Não & & \\
Fumante ou ex-fumante & 35 & 20,2 \\
Sim & 138 & 79,8 \\
Não & & \\
Diabetes & 13 & 7,5 \\
Sim & 160 & 92,5 \\
Não & & \\
Doença cardíaca & 10 & 5,8 \\
Sim & 163 & 94,2 \\
Não & & \\
Uso de medicação & 49 & 28,3 \\
Sim & 124 & 71,7 \\
Não & $\mathbf{1 7 3}$ & $\mathbf{1 0 0 , 0}$ \\
Total & & \\
\hline
\end{tabular}

A Tabela 3 mostra os resultados da análise bivariada. Foi verificada associação estatisticamente significativa entre periodontite e sexo masculino $(p=0,009)$, faixa etária mais avançada $(p<0,001)$, hipertensão arterial $(p=0,001)$, tabagismo ou histórico de tabagismo $(p=0,034)$ e diabetes $(p=0,015)$. A prevalência de periodontite foi maior entre homens $(45,5 \%)$ em comparação com mulheres; entre pacientes das faixas etárias mais avançadas, como 50-59 anos (88,2\%) e 60 anos ou mais (80\%) em comparação com faixas etárias menores; entre pacientes hipertensos $(65,4 \%)$, fumantes ou ex-fumantes $(51,4 \%)$ e diabéticos $(69,2 \%)$ em comparação com aqueles que eram não hipertensos, não fumantes ou ex-fumantes e não diabéticos. 
Tabela 3. Análise de associação entre diagnóstico periodontal, condições sistêmicas, tabagismo e uso de medicação

\begin{tabular}{|c|c|c|c|c|c|c|c|}
\hline \multicolumn{8}{|c|}{ Diagnóstico periodontal } \\
\hline \multirow{2}{*}{ Variáveis } & \multicolumn{2}{|c|}{ Gengivite } & \multicolumn{2}{|c|}{ Periodontite } & \multicolumn{2}{|c|}{ Total } & \multirow[t]{2}{*}{ p-valor } \\
\hline & n & $\%$ & n & $\%$ & $\mathbf{n}$ & $\%$ & \\
\hline Sexo & & & & & & & $0,009(1) *$ \\
\hline Feminino & 62 & 73,8 & 22 & 26,2 & 84 & 100,0 & \\
\hline Masculino & 48 & 54,5 & 40 & 45,5 & 88 & 100,0 & \\
\hline Faixa etária & & & & & & & $0,001(2) *$ \\
\hline$\leq 19$ anos & 26 & 100,0 & 0 & 0,0 & 26 & 100,0 & \\
\hline 20-29 anos & 42 & 84,0 & 8 & 16,0 & 50 & 100,0 & \\
\hline 30-39 anos & 24 & 58,5 & 17 & 41,5 & 41 & 100,0 & \\
\hline 40-49 anos & 15 & 45,5 & 18 & 54,5 & 33 & 100,0 & \\
\hline 50-59 anos & 2 & 11,8 & 15 & 88,2 & 17 & 100,0 & \\
\hline$\geq 60$ anos & 1 & 20,0 & 4 & 80,0 & 5 & 100,0 & \\
\hline Hipertensão & & & & & & & $0,001(1) *$ \\
\hline Sim & 9 & 34,6 & 17 & 65,4 & 26 & 100,0 & \\
\hline Não & 101 & 69,2 & 45 & 30,8 & 146 & 100,0 & \\
\hline Diabetes & & & & & & & $0,015(1) *$ \\
\hline Sim & 4 & 30,8 & 9 & 69,2 & 13 & 100,0 & \\
\hline Não & 106 & 66,7 & 53 & 33,3 & 159 & 100,0 & \\
\hline Doença cardíaca & & & & & & & $0,344(1)$ \\
\hline Sim & 5 & 50,0 & 5 & 50,0 & 10 & 100,0 & \\
\hline Não & 105 & 64,8 & 57 & 35,2 & 162 & 100,0 & \\
\hline Fumante ou ex-fumante & & & & & & & $0,034(1) *$ \\
\hline Sim & 17 & 48,6 & 18 & 51,4 & 35 & 100,0 & \\
\hline Não & 93 & 67,9 & 44 & 32,1 & 137 & 100,0 & \\
\hline Uso de medicação & & & & & & & $0,816^{(1)}$ \\
\hline Sim & 32 & 65,3 & 17 & 34,7 & 49 & 100,0 & \\
\hline Não & 78 & 63,4 & 45 & 36,6 & 123 & 100,0 & \\
\hline
\end{tabular}

(1)Teste qui-quadrado de Pearson. (2)Teste exato de Fisher. ${ }^{*} \mathrm{p}<0,05$.

De acordo com a Tabela 4, foi verificada associação estatisticamente significativa entre menor quantidade de dentes presentes, pacientes com faixa etária mais avançada $(p<0,001)$, hipertensos $(p=0,001)$, diabéticos $(p=0,011)$, cardiopatas $(p=0,002)$ e fumantes ou ex-fumantes $(p=0,015)$. Uma quantidade de dentes igual ou menor do que 10 foi observada com maior frequência entre pacientes das faixas etárias mais avançadas, como com idade igual ou superior a 60 anos (80\%), quando comparados com faixas etárias menores, e entre hipertensos $(15,4 \%)$, diabéticos $(15,4 \%)$, cardiopatas $(20 \%)$ e fumantes ou ex-fumantes $(20 \%)$, em comparação com aqueles que não eram hipertensos, diabéticos, cardiopatas, fumantes ou ex-fumantes.

Tabela 4. Análise de associação entre quantidade de dentes presentes, condições sistêmicas, tabagismo e uso de medicação

\begin{tabular}{|c|c|c|c|c|c|c|c|c|c|}
\hline \multirow{3}{*}{ Variáveis } & \multicolumn{8}{|c|}{ № de dentes presentes } & \multirow{3}{*}{ p-valor } \\
\hline & \multicolumn{2}{|c|}{ 0-10 } & \multicolumn{2}{|c|}{ 11-20 } & \multicolumn{2}{|c|}{$>20$} & \multicolumn{2}{|c|}{ Total } & \\
\hline & $\mathbf{n}$ & $\%$ & $\mathbf{n}$ & $\%$ & $\mathbf{n}$ & $\%$ & $\mathbf{n}$ & $\%$ & \\
\hline Feminino & 7 & 8,2 & 11 & 12,9 & 67 & 78,8 & 85 & 100,0 & \\
\hline Masculino & 2 & 2,3 & 19 & 21,6 & 67 & 76,1 & 88 & 100,0 & \\
\hline$\leq 19$ anos & 0 & 0,0 & 0 & 0,0 & 27 & 100,0 & 27 & 100,0 & \\
\hline 20-29 anos & 0 & 0,0 & 2 & 4,0 & 48 & 96,0 & 50 & 100,0 & \\
\hline 30-39 anos & 1 & 2,4 & 6 & 14,6 & 34 & 82,9 & 41 & 100,0 & \\
\hline
\end{tabular}


Tabela 4. Continuação...

\begin{tabular}{|c|c|c|c|c|c|c|c|c|c|}
\hline \multirow{3}{*}{ Variáveis } & \multicolumn{8}{|c|}{ № de dentes presentes } & \multirow{3}{*}{ p-valor } \\
\hline & \multicolumn{2}{|c|}{ 0-10 } & \multicolumn{2}{|c|}{$11-20$} & \multicolumn{2}{|c|}{$>20$} & \multicolumn{2}{|c|}{ Total } & \\
\hline & $\mathbf{n}$ & $\%$ & $\mathbf{n}$ & $\%$ & $\mathbf{n}$ & $\%$ & $\mathbf{n}$ & $\%$ & \\
\hline 40-49 anos & 2 & 6,1 & 14 & 42,4 & 17 & 51,5 & 33 & 100,0 & \\
\hline 50-59 anos & 2 & 11,8 & 7 & 41,2 & 8 & 47,1 & 17 & 100,0 & \\
\hline$\geq 60$ anos & 4 & 80,0 & 1 & 20,0 & 0 & 0,0 & 5 & 100,0 & \\
\hline Hipertensão & & & & & & & & & $0,001(2) *$ \\
\hline Sim & 4 & 15,4 & 9 & 34,6 & 13 & 50,0 & 26 & 100,0 & \\
\hline Não & 5 & 3,4 & 21 & 14,3 & 121 & 82,3 & 147 & 100,0 & \\
\hline Diabetes & & & & & & & & & $0,011^{(2) *}$ \\
\hline Sim & 2 & 15,4 & 5 & 38,5 & 6 & 46,2 & 13 & 100,0 & \\
\hline Não & 7 & 4,4 & 25 & 15,6 & 128 & 80,0 & 160 & 100,0 & \\
\hline Doença cardíaca & & & & & & & & & $0,002^{(2) *}$ \\
\hline Sim & 2 & 20,0 & 5 & 50,0 & 3 & 30,0 & 10 & 100,0 & \\
\hline Não & 7 & 4,3 & 25 & 15,3 & 131 & 80,4 & 163 & 100,0 & \\
\hline Fumante ou ex-fumante & & & & & & & & & $0,015(2) *$ \\
\hline Sim & 5 & 14,3 & 8 & 22,9 & 22 & 62,9 & 35 & 100,0 & \\
\hline Não & 4 & 2,9 & 22 & 15,9 & 112 & 81,2 & 138 & 100,0 & \\
\hline Uso de medicação & & & & & & & & & $0,535^{(2)}$ \\
\hline Sim & 4 & 8,2 & 8 & 16,3 & 37 & 75,5 & 49 & 100,0 & \\
\hline Não & 5 & 4,0 & 22 & 17,7 & 97 & 78,2 & 124 & 100,0 & \\
\hline
\end{tabular}

(2)Teste exato de Fisher. ${ }^{*} \mathrm{p}<0,05$.

\section{DISCUSSÃO}

A idade e o sexo são fatores de risco não modificáveis tanto para doenças periodontais como para doenças cardiovasculares ${ }^{7}$. A presente pesquisa apresentou maior incidência de doenças periodontais em pacientes idosos, assim como foi observado no estudo de Almeida et al. ${ }^{8}$, e maior predileção de doenças para o sexo masculino, resultado encontrado por Goulart et al. ${ }^{9}$, mas diferente de Almeida et al. ${ }^{8}$, cujo estudo indicou que a maior incidência era em mulheres.

Os patógenos periodontais podem destruir o epitélio da bolsa periodontal, permitindo, assim, a entrada de endotoxinas e exotoxinas nocivas na corrente sanguínea ${ }^{10}$. Também ocorre invasão direta da parede do vaso por patógenos orais, desencadeando resposta inflamatória que conduz à disfunção endotelial. Por exemplo, hábitos de higiene oral ou procedimentos odontológicos, ao gerarem bacteremia transitória assintomática, podem levar microrganismos a ter contato direto com a circulação e, consequentemente, com o endotélio coronariano. Portanto, a DP conduz a um aumento local de mediadores inflamatórios que promovem inflamação crônica endotelial, sugerindo que o processo inflamatório possa contribuir para vasoespasmo e trombose. Desse modo, quanto mais disseminada a infecção pelos tecidos de sustentação dentária, maior será a área de exposição ao sistema vascular ${ }^{11}$. Apesar disso, este estudo não verificou associação significativa entre DP e doenças cardíacas.

Com relação ao histórico de tabagismo, a prevalência da DP foi maior entre pacientes fumantes ou ex-fumantes $(51,4 \%)$ em comparação com os não fumantes ou ex-fumantes. Achados como esses também foram encontrados em outros estudos ${ }^{12,13}$. 0 tabagismo é um forte fator de risco para as doenças periodontais. Além disso, é sugerido que os mecanismos pelos quais o tabagismo favorece a destruição periodontal estão relacionados a uma menor regulação dos fatores da inflamação ${ }^{14}$. Em geral, foi relatado que a modulação do tabagismo na destruição óssea da DP pode envolver níveis reduzidos de fatores anti-inflamatórios e antirreabsortivos, como IL-10 e osteoprotegerina, respectivamente, e também pode envolver altos níveis de citocinas pró-inflamatórias, como IL - 6 e INF- $\gamma^{15}$. 
Já a diabetes mellitus (DM) é considerada uma síndrome clínica, caracterizada por hiperglicemia, causada por deficiência herdada e/ou adquirida na produção e/ou ação de insulina ${ }^{15}$. Geralmente, a má higiene bucal, uma longa história de diabetes mellitus e um pobre controle metabólico estão associados à maior gravidade da DP. A associação entre as duas doenças pode ser por causa de numerosos fenômenos fisiopatológicos encontrados na DM, tais como: resistência diminuída, alterações vasculares, alteração da microflora bucal e metabolismo anormal do colágeno ${ }^{16}$. Delgado-Pérez et al. ${ }^{17}$ constataram que pacientes portadores de diabetes mellitus apresentaram uma porcentagem maior de perdas dentárias, assim como este estudo. Simultaneamente, esses pacientes apresentaram uma prevalência maior de DP em comparação com pacientes não diabéticos.

Vem sendo demonstrado que os pacientes diabéticos apresentam maior perda óssea, maior perda de inserção, maior profundidade de sondagem e maior inflamação gengival do que os que não possuem DM em uma mesma faixa etária ${ }^{18}$. Ainda assim, Polak e Shapira ${ }^{19}$ não observaram uma associação entre diabetes e DP, o que sugere a necessidade de investigações adicionais para confirmar a ligação potencial entre esses dois distúrbios.

Há na literatura diversos estudos que investigam a possível associação entre as alterações ou doenças sistêmicas e a DP3-20. Alguns indicam associação positiva, enquanto outros afirmam não existir associação. Por esse motivo, são necessários mais estudos para que se possa considerar a DP como um fator de risco para essas condições. É preciso destacar ainda a necessidade de tratamento multidisciplinar pelos profissionais da saúde para melhor diagnóstico de condições sistêmicas e doenças periodontais, enfatizando a necessidade da manutenção da saúde bucal para prevenção e controle dessas doenças.

\section{CONCLUSÃO}

Foi verificada, neste estudo, associação estatisticamente significativa $(\mathrm{p}<0,05)$ entre periodontite e faixa etária $\geq 60$ anos, hipertensão arterial, tabagismo ou histórico de tabagismo e diabetes. Entretanto, por se tratar de um estudo de caráter retrospectivo, não houve acompanhamento longitudinal dos pacientes atendidos e, portanto, não foram observadas as causas e os mecanismos fisiopatológicos que justificassem essas associações. Em vista disso, há a necessidade de mais estudos para que se possa afirmar, com exatidão, essas correlações.

\section{REFERÊNCIAS}

1. Steffens JP, Marcantonio RAC. Classificação das doenças e condições periodontais e peri-implantares 2018: guia prático e pontos-chave. Rev Odontol UNESP. 2018 Jul-Ago.;47(4):189-97. http://dx.doi.org/10.1590/1807-2577.04704.

2. Hajishengallis G. Periodontitis: from microbial immune subversion to systemic inflammation. Nat Rev Immunol. 2015 Jan;15(1):30-44. http://dx.doi.org/10.1038/nri3785. PMid:25534621.

3. Louro PM, Fiori HH, Louro P Fo, Steibel J, Fiori RM. Doença periodontal na gravidez e baixo peso ao nascer. J Pediatr. 2001;77(1):23-8. http://dx.doi.org/10.1590/S0021-75572001000100008. PMid:14647615.

4. Kinane DF, Marshall GJ. Periodontal manifestations of systemic disease. Aust Dent J. 2001 Mar;46(1):212. http://dx.doi.org/10.1111/j.1834-7819.2001.tb00267.x. PMid:11355236.

5. Tettamanti L, Lauritano D, Nardone M, Gargari M, Silvestre-Rangil J, Gavoglio P, et al. Pregnancy and periodontal disease: does exist a two-way relationship? Oral Implantol. 2017 Sep;10(2):112-8. http://dx.doi.org/10.11138/orl/2017.10.2.112. PMid:29876036.

6. Gusmão ES, dos Santos RL, Silveira RCJ, de Souza EHA. Avaliação clínica e sistêmica em pacientes que procuram tratamento periodontal. Rev Odonto Ciênc. 2005 Jul-Set;20(49):199-203. 
7. Górski B, Nargiello E, Opolski G, Ganowicz E, Górska R. The association between dental status and systemic lipid profile and inflammatory mediators in patients after myocardial infarction. Adv Clin Exp Med. 2016 Jul-Aug;25(4):625-30. http://dx.doi.org/10.17219/acem/62937. PMid:27629835.

8. Almeida BB, Ferreira ACR, Queiroz APG, Dornelas GN, Coelho MMST. Condições periodontais em portadores de diabetes mellitus atendidos no centro de referencia sul fluminense de diabetes e hipertensão de Vassouras-RJ. Braz J Periodontol. 2015;25(4):14-23.

9. Goulart AC, Armani F, Arap AM, Nejm T, Andrade JB, Bufarah HB, et al. Relação entre doença periodontal e fatores de risco cardiovascular em brasileiros jovens e de meia-idade. Estudo transversal. Sao Paulo Med J. 2017 Jun;135(3):226-33. http://dx.doi.org/10.1590/1516-3180.2016.0357300117. PMid:28746658.

10. Sudhakara P, Gupta A, Bhardwaj A, Wilson A. Oral dysbiotic communities and their implications in systemic diseases. Dent. J. 2018 Apr;6(2):10. http://dx.doi.org/10.3390/dj6020010. PMid:29659479.

11. Paizan ML, Martin JFV. Associação entre doença periodontal, doença cardiovascular e hipertensão arterial. Rev Bras Hipertens. 2009 Jul-Set;16(3):183-5.

12. Monteiro-da-Silva F, Sampaio-Maia B, Pereira ML, Araújo R. Characterization of the oral fungal microbiota in smokers and nonsmokers. Eur J Oral Sci. 2013 Apr;121(2):132-5. http://dx.doi.org/10.1111/eos.12030. PMid:23489903.

13. Bernardes V, Ferres MO, Lopes W Jr. 0 tabagismo e as doenças periodontais. Rev Faculdade Odontol Lins. 2013;23(1):37-45. http://dx.doi.org/10.15600/2238-1236/fol.v23n1p37-45.

14. César-Neto JB, Rosa EF, Pannuti CM, Romito GA. Smoking and periodontal tissues: a review. Braz Oral Res. 2012 Jan;26(spe1 Suppl 1):25-31. http://dx.doi.org/10.1590/S1806-83242012000700005. PMid:23318741.

15. Vernillo AT. Dental considerations for the treatment of patients with diabetes mellitus. J Am Dent Assoc. 2003 Oct;134(Spec No):24S-33S. http://dx.doi.org/10.14219/jada.archive.2003.0366. PMid:18196670.

16. César-Neto JB, Duarte PM, de Oliveira MC, Tambeli CH, Sallum EA, Nociti FH Jr. Smoking modulates IL6:IL-10 and RANKL:OPG ratios in the periodontal tissues. J Periodontal Res. 2007 Apr;42(2):184-91. http://dx.doi.org/10.1111/j.1600-0765.2006.00934.x. PMid:17305878.

17. Delgado-Pérez VJ, De La Rosa-Santillana R, Márquez-Corona ML, Ávila-Burgos L, Islas-Granillo H, MinayaSánchez M, et al. Diabetes or hypertension as risk indicators for missing teeth experience: An exploratory study in a sample of Mexican adults. Niger J Clin Pract. 2017 Oct;20(10):1335-41. http://dx.doi.org/10.4103/njcp.njcp_52_17. PMid:29192641.

18. Monteiro AMA, Monteiro CSPA, Sassi MS, Moreira DC, Pereira RP, Gusmão JMR, et al. Prevalência da doença periodontal em diabéticos controlados. Perionews. 2014 Set-Out;8(5):475-80.

19. Polak D, Shapira L. An update on the evidence for pathogenic mechanisms that may link periodontitis and diabetes. J Clin Periodontol. 2018 Feb;45(2):150-66. http://dx.doi.org/10.1111/jcpe.12803. PMid:29280184.

20. Rengo G, Pagano G, Paolillo S, Lucia C, Femminella GD, Liccardo D, et al. Impact of diabetes mellitus on lymphocyte GRK2 protein levels in patients with heart failure. Eur J Clin Invest. 2015 Feb;45(2):187-95. http://dx.doi.org/10.1111/eci.12395. PMid:25545706.

\section{CONFLITOS DE INTERESSE}

Os autores declaram não haver conflitos de interesse.

\section{*AUTOR PARA CORRESPONDÊNCIA}

Rachel de Queiroz Ferreira Rodrigues, UFCG - Universidade Federal de Campina Grande, Unidade Acadêmica de Ciências Biológicas, Avenida Universitária, s/n, Santa Cecilia, 58708-110 Patos - PB, Brasil, e-mail: rachelperio@gmail.com 